\title{
Arqueología marítima en la Patagonia: el proyecto HMS Swift (Argentina)
}

Dolores Elkin | Consejo Nacional de Investigaciones Científicas y Técnicas (CONICET)

URL de la contribución <www.iaph.es/revistaph/index.php/revistaph/article/view/3748>

\section{RESUMEN}

El trabajo sintetiza las principales líneas de investigación arqueológica e interdisciplinaria desarrolladas en el proyecto HMS Swift, referido a una nave de guerra inglesa naufragada en 1770 en la ría Deseado (actual provincia de Santa Cruz, Argentina). El trabajo fue llevado a cabo por el equipo del Programa de Arqueología Subacuática (PROAS) del Instituto Nacional de Antropología y Pensamiento Latinoamericano, organismo dependiente del Ministerio de Cultura de la República Argentina.

Los temas de estudio tratados comprenden aspectos constructivos del barco, la relación entre los artefactos transportados a bordo y las distintas jerarquías sociales representadas en la tripulación, enmarcado a su vez en los avances tecnológicos que caracterizaron al siglo XVIII en Europa, la vida a bordo de la nave, y los procesos de formación del sitio ocurridos desde el hundimiento del barco hasta la actualidad. También se hace referencia a una línea de investigación no prevista inicialmente, consistente en el estudio de restos humanos.

Tras la presentación de los resultados surgidos de la investigación científica, se hace mención a otras facetas del proyecto Swift, consistentes en la relación entre éste y la comunidad, las posibilidades de desarrollo turístico en torno a la colección arqueológica proveniente del pecio y el grado de valoración patrimonial pública que posee este sitio.

\section{Palabras clave}

Argentina | Arqueología subacuática | Excavaciones arqueológicas | HMS Swift | Intervenciones | Naufragio | Patagonia (Argentina) | Patrimonio Arqueológico Subacuático | Pecios | Santa Cruz (Argentina) | Siglo XVIII | 


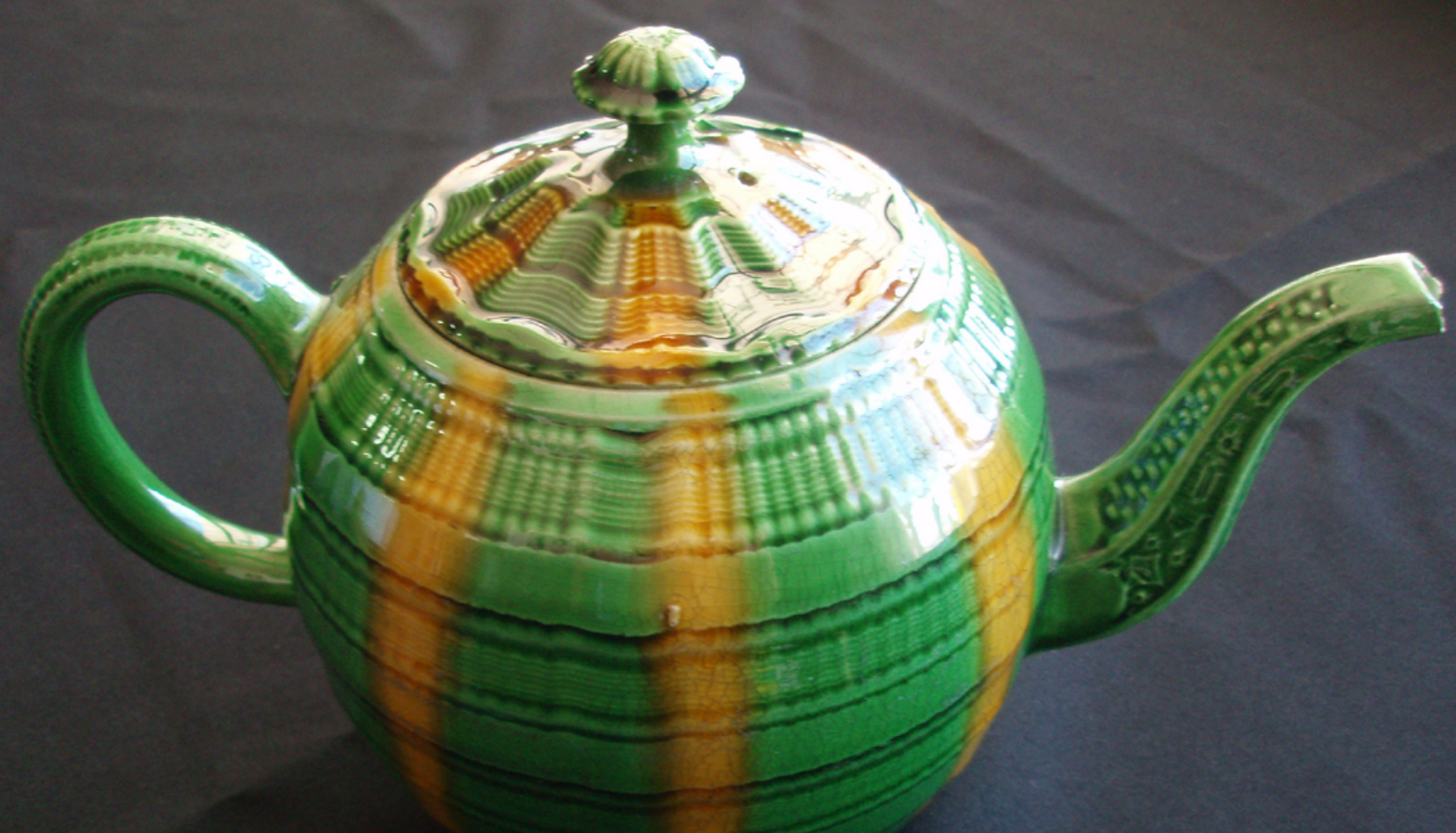

Tetera de cerámica decorada hallada en un sector del pecio Swift vinculado a los oficiales | foto todas las imágenes que ilustran este artículo forman parte del Programa de Arqueología Subacuática-INAPL, si no se indica lo contrario 

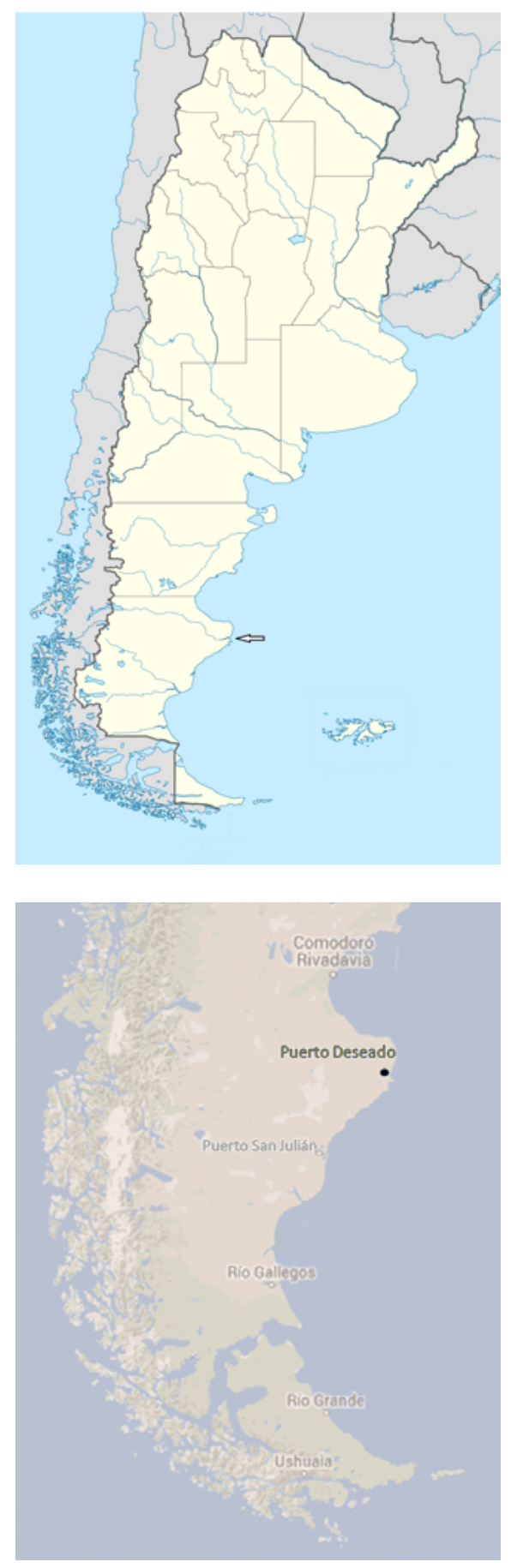

1. Mapa de la República Argentina y detalle de la zona geográfica donde se ubica Puerto Deseado y otras localidades mencionadas en el texto | mapas elaboración propia a partir de cartografía del Instituto Geográfico Nacional de Argentina y Google maps

\section{INTRODUCCIÓN}

Hacia las últimas décadas del XVIII y ya concluida la Guerra de los Siete Años, las islas Malvinas, ubicadas en el océano Atlántico suroccidental, eran disputadas por tres potencias marítimas europeas: España, Francia e Inglaterra. Estas dos últimas las denominaban Malouines y Falklands respectivamente, e incluso ya habían construido sendos asentamientos en las islas como base de pequeñas escuadras navales.

Fue en este contexto que, a principios de marzo de 1770, partió de la base de Puerto Egmont una de las naves inglesas con el propósito de realizar exploraciones por la región. Se trataba de la corbeta de guerra HMS Swift, artillada con 14 cañones de hierro de 6 libras y 14 pedreros. Su capitán era George Farmer y llevaba cerca de un centenar de tripulantes.

Según lo expresado en documentos históricos, un viento intenso y persistente condujo a la nave hacia la costa continental de La Patagonia, entre las actuales localidades de Puerto San Julián y Puerto Deseado (imagen 1). El capitán Farmer decidió dirigirse a este último sitio, ya conocido como un buen puerto natural. Allí la nave estaría a buen reparo y la tripulación podría descansar y recuperarse tras los penosos días de mal tiempo (GOWER, 1803).

Sin embargo, a poco de ingresar en la ría Deseado la nave encalló sucesivamente en dos rocas ocultas por la marea alta. En la segunda oportunidad, tras varias horas de maniobras y esfuerzos desesperados por evitar el naufragio apenas el agua comenzó a bajar, el barco se deslizó repentinamente hacia popa y segundos más tarde desaparecía por completo en esta remota región.

A excepción de solo tres personas, el resto de la tripulación pudo llegar a tierra firme utilizando los botes auxiliares de la corbeta. Algunas semanas después, gracias al heroico viaje hasta Puerto Egmont realizado por seis voluntarios en uno de esos botes -acondicionado lo mejor posible para la larga y riesgosa travesía- todos los sobrevivientes fueron rescatados por otra nave de la escuadra inglesa, la Favourite.

La corbeta Swift y casi todo lo que llevaba a bordo en su último viaje permanecerían en el lecho de la ría Deseado durante más de dos siglos. La existencia del naufragio se conoció públicamente recién en el año 1975, cuando un australiano de nombre Patrick Gower, descendiente del teniente de la Swift Erasmus Gower, viajó a Puerto Deseado llevando consigo un diario escrito por su ancestro donde constaba el lugar del accidente (GOWER, 1803).

En base a este testimonio fue que pocos años después un grupo de entusiastas buzos deseadenses tomó la iniciativa de iniciar una búsqueda sistemática del pecio. Contaban con el auspicio del club náutico local y del 
Museo Naval de la Nación, aunque carecían de elementos básicos como un compresor de buceo en Puerto Deseado, por lo que debían recorrer cientos de kilómetros solo para cargar de aire los botellones. A esta meritoria actitud debe agregarse otra aun más destacable: desde un principio los jóvenes acordaron que, en caso de encontrar el pecio, todos los materiales formarían parte del patrimonio público de la pequeña localidad donde residían.

Finalmente, en febrero de 1982 se produjo el esperado hallazgo, resultando llamativo el excelente estado de preservación de todos los restos, tanto la estructura del barco en sí como de los objetos que comenzaban a extraerse. Ello se debía fundamentalmente a la combinación de la baja temperatura del agua con la capa de sedimento fino y compacto que recubre el sitio, condiciones que reducen las probabilidades de desarrollo de distintos tipos de organismos que pueden dañar los materiales. Sumado a ello, hay que tener en cuenta que el proceso de naufragio de la corbeta Swift fue relativamente poco traumático, a diferencia de lo que sucede cuando una nave se hunde en combate, por un incendio, o tras impactar violentamente contra escollos rocosos o arrecifes. Todo esto permitió que el casco y la mayoría de los objetos contenidos en su interior llegaran al lecho marino prácticamente sin romperse, aunque la arboladura y otros elementos de la parte superior de la nave fueron perdiéndose con el paso del tiempo.

Tras el importante hallazgo, el sitio Swift sería declarado patrimonio histórico de la provincia de Santa Cruz, creándose también en Puerto Deseado un museo destinado a albergar toda la colección arqueológica procedente del naufragio. El museo lleva el nombre de Mario Brozoski, en honor a uno de los descubridores de la corbeta Swift, y quien falleciera poco tiempo después.

En la década subsiguiente al descubrimiento se realizaron diversos tipos de intervenciones en el sitio, principalmente a cargo del Grupo de Trabajo de Patrimonio Subacuático (GTPS) del Consejo Internacional de Monumentos y Sitios (ICOMOS). Este grupo estaba conformado en su mayor parte por arquitectos-buceadores, muy comprometidos con la preservación de este sitio a pesar de carecer de formación en arqueología.

En 1997, a petición de las autoridades provinciales santacruceñas a través del Museo Brozoski, comenzó la investigación científica de los restos de la corbeta Swift, esta vez a cargo del recientemente creado equipo de arqueología subacuática del Instituto Nacional de Antropología y Pensamiento Latinoamericano.

El proyecto Swift marcaría un hito en la historia de la disciplina arqueológica en Argentina, ya que se trató de la primera investigación focalizada en vestigios culturales sumergidos dirigida y protagonizada por profesionales graduados en Arqueología, y que además habían recibido capacitación en buceo. 


\section{PRINCIPALES LÍNEAS DE INVESTIGACIÓN, MATERIALES Y MÉTODOS}

En el diseño de investigación original (ELKIN, 1997) se propusieron diversas líneas y temas de trabajo, acompañados de sus respectivas hipótesis, todo lo cual debió ajustarse a variables tales como la seguridad de los buceadores -esto en primer lugar-, la capacidad operativa del equipo de investigación, tanto en relación con los recursos humanos como a lo económicos, al equipamiento disponible y la infraestructura y personal del Museo Brozoski, el cual estaría a cargo de la conservación y guarda de todos los materiales eventualmente extraídos del sitio.

Las actividades de buceo estarían desde luego sujetas a las condiciones hidrometeorológicas imperantes en el sitio, caracterizadas por fuertes corrientes, gran amplitud de marea, baja temperatura del agua y escasa visibilidad debido a las finas partículas de sedimento en suspensión permanentemente presentes en la ría.

Los relevamientos iniciales se centraron en la elaboración de un plano general del sitio. La metodología implementada para los trabajos de planimetría consistió en mediciones con cinta métrica a partir de una línea de referencia o línea de base, registrando progresivamente todas las cuadernas que sobresalían del sedimento -especialmente a lo largo de la banda de estribor-y que conformaban el perímetro de la zona principal de trabajo. También se registró la ubicación de algunos elementos destacados, como cañones y anclas (imagen 2).

La excavación se iniciaría algunas temporadas después, una vez seleccionados los sectores del pecio destinados a ello en función de los temas y preguntas de investigación planteados. Por distintas razones se consideró apropiado excavar en base a niveles artificiales de $40 \mathrm{~cm}$ de espesor, pero teniendo en cuenta las divisiones internas de la nave tales como camarotes y otros recintos.

También fue necesario consagrar los primeros años de trabajo a la mejor comprensión de aspectos históricos vinculados con la corbeta Swift. En este último sentido, además de analizar exhaustivamente el diario escrito por Gower (GOWER, 1803), se comenzaron a localizar y recopilar distintos tipos de fuentes primarias en archivos británicos, tales como los planos del barco (NMM ZAZ 4672; NMM ZAZ 4724), la correspondencia mantenida entre los oficiales de la Swift y varias dependencias de la Royal Navy (ADM, 1/1789), e incluso la corte marcial que tuvo lugar debido a la pérdida de la nave (ADM, 1/5304).

A pesar de disponer de este tipo de documentación histórica y considerarla de indiscutible utilidad en la investigación para el tratamiento de temas como el recién señalado, siempre se puso el foco en la evidencia material, que es 


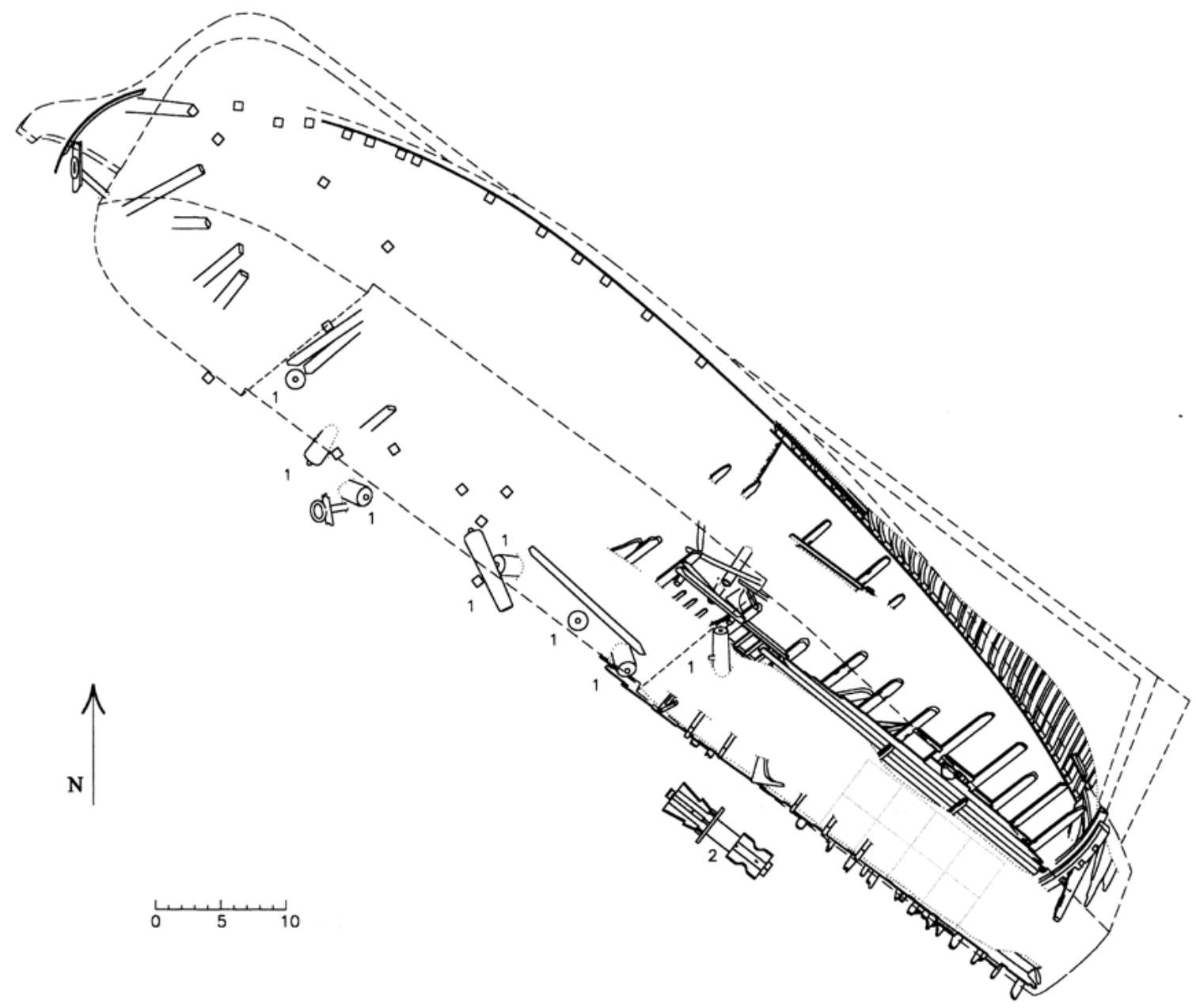

el objeto de estudio de la arqueología propiamente dicha. Consideramos que 2. Plano inicial del sitio Swift. 1. Cañones; 2. Cabrestante (escala en pies) | dibujo C. Muray (Programa de Arqueología Subacuática-INAPL) ello debe tenerse presente aun, o quizás especialmente, cuando el tema tratado corresponde a una época para la cual se cuenta con información escrita ya que se corre el riesgo de sesgar u omitir aspectos de la investigación arqueológica en virtud de lo que "ya se sabe" por las fuentes históricas. A partir de los restos arqueológicos que conforman el sitio Swift se esperaba poder tratar aspectos constructivos del barco, la relación entre los artefactos transportados a bordo y las distintas jerarquías sociales representadas en la tripulación, enmarcado a su vez en los avances tecnológicos que caracterizaron al siglo XVIII en Europa, la vida a bordo de la nave, y los procesos de formación del sitio ocurridos desde el hundimiento del barco hasta la actualidad.

El conocimiento generado en dichas líneas de estudio fue volcado de manera exhaustiva en una obra de compendio publicada hace pocos años 
(ELKIN; MURRAY; BASTIDA et ál., 2011), y previamente a ello se ha ido presentando de manera parcial en relación con diversos temas (por ejemplo, BASTIDA; GROSSO; ELKIN, 2008; BASTIDA; ELKIN; GROSSO, 2010; DELLINO-MUSGRAVE, 2006; ELKIN, 2003; ELKIN; ARGÜESO, 2010; ELKIN; ARGÜESO; GROSSO et ál., 2007; MURRAY; ELKIN; VAINSTUB, 2002-2003; CIARLO, 2011; GROSSO, 2008; 2011). A continuación se brinda una síntesis de la información disponible hasta la fecha.

\section{RESULTADOS}

\section{Construcción del barco}

Al observar los planos originales de la Swift resulta evidente una contradicción significativa: Uno de ellos -el plano de cubiertas- ilustra la nave arbolada con dos palos (NMM ZAZ 4724), en tanto que otro -el llamado plano de líneas- lo hace con tres (NMM ZAZ 4672). En consecuencia, uno de los primeros interrogantes a dilucidar fue cómo era realmente el barco al momento del naufragio y cómo podía explicarse la discordancia entre dos planos oficiales. Una de las respuestas surgió a poco de comenzar los buceos en el sitio, al identificarse la base del palo mesana, es decir el que se ubica más a popa en un barco arbolado con tres palos. Lo que no ha podido esclarecerse de manera fehaciente es por qué entonces uno de los planos muestra a la Swift con dos palos. Un indicio interesante lo provee uno de los documentos históricos disponibles (NMM ADM 180/3) el cual consigna una modificación efectuada al casco, de considerable costo. Es posible que la misma haya incluido el agregado de un tercer palo, aunque ese dato no está consignado.

Cerca de la zona de proa-babor pero fuera del perímetro del casco se halló una bomba aspirante portátil realizada en plomo (imagen 3). Como puede apreciarse en la fotografía, una de las caras del receptáculo posee la inscripción G.R. ${ }^{3}$ 1769. Las iniciales y el número 3 corresponden al rey Jorge (George Rex) tercero, y la fecha coincide con la de trabajos de mantenimiento realizados a la Swift en el arsenal de Deptford (NMM ADM 180/3 Swift 1762-1769), por lo que probablemente fue colocada en ese momento.

\section{Tecnología y relación entre cultura material y jerarquías sociales}

En términos generales, esta línea de investigación se basa en una hipótesis de sentido común: que los oficiales utilizaban elementos de mayor calidad en tanto que con los marineros comunes sucedía el caso inverso. Los artefactos de buena calidad, a su vez, podrían reflejar los progresos tecnológicos que caracterizaron al siglo XVIII europeo. 
En virtud de lo observado en la colección arqueológica proveniente del sitio Swift puede decirse que la mayoría de los artefactos asociados a los oficiales (hallados en las dependencias de popa en la cubierta principal de la nave) muestran una buena calidad de manufactura, e incluyen cerámica inglesa y porcelana china (imágenes de portada y 4). Otros objetos que también fueron hallados en el sector de la nave ocupado por los oficiales y poseen buena calidad están realizados en metal y en vidrio (imágenes 5 y 6 ).

En cuanto a las técnicas de manufactura empleadas, una de las más destacadas es la de la tetera ilustrada en la imagen de portada, en especial por la decoración geométrica del cuerpo y de la tapa, que requiere el empleo de un torno mecánico especial que efectúa movimientos sincronizados en distintas direcciones. Algunos autores consideran que esta técnica decorativa parece haberse utilizado en pocos lugares de Inglaterra, y recién a partir de la década de 1760 (HAWKINS, 1999). Estos tornos eran costosos y pocas fábricas disponían de ellos, así como de los torneros especializados para operarlos. Podría decirse entonces que esta tetera ilustra avances tecnológicos de la época, al menos en cuanto a la producción cerámica.

Independientemente de la calidad de manufactura y el grado de estatus social que ciertos objetos podían representar, también pueden apreciarse otros aspectos de la cultura material asociada al sitio Swift. Por ejemplo,

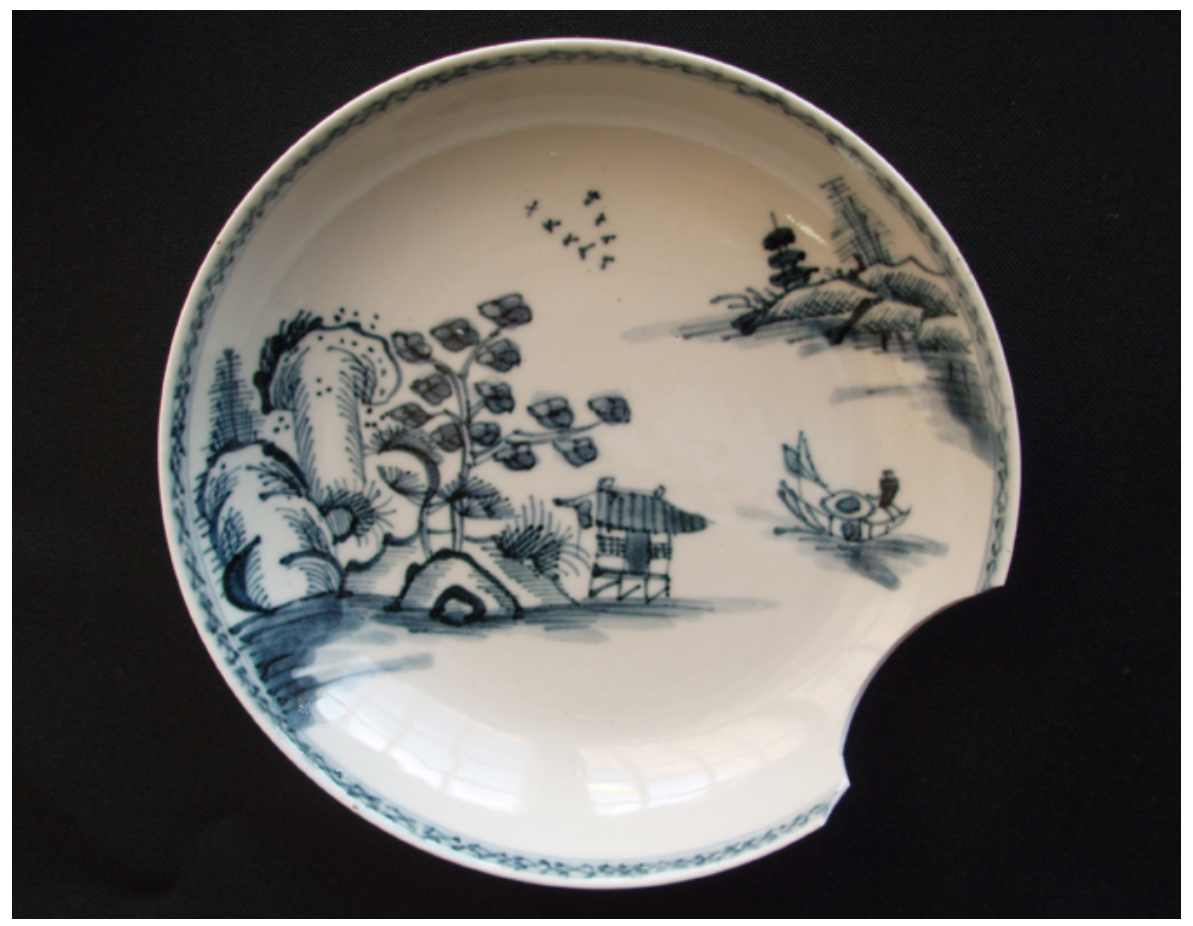

4,5 y 6 . Distintos objetos hallados en sectores del pecio Swift vinculados a los oficiales de la nave: plato de porcelana oriental, cuchara de peltre con sello del fabricante y tapón de vidrio facetado
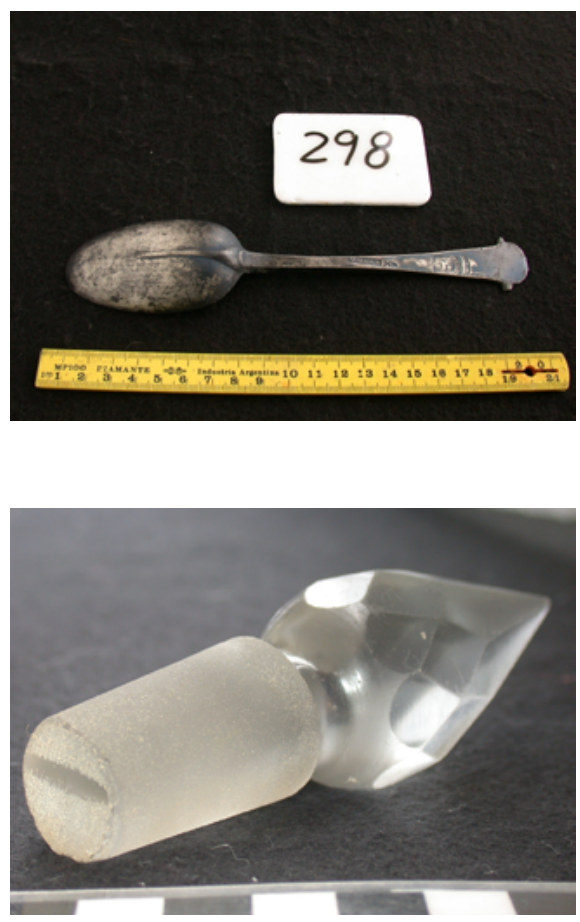
muchas de las piezas halladas en el sector ocupado por los oficiales corresponden al servicio del té, lo cual refleja las costumbres y tradiciones de su tierra natal y de lo que podría llamarse un sentido de Britishness (DELLINOMUSGRAVE, 2005).

Sabemos menos acerca del tipo de elementos asociados a los marineros comunes, aunque se hallaron platos de madera en el área de la cocina que podrían haber pertenecido a éstos, no siendo posible corroborarlo. Se espera, en un futuro cercano, poder avanzar con la excavación de los sectores de uso común en la zona central y de proa del barco, los cuales podrían brindar evidencia arqueológica de utilidad para evaluar este tema.

\section{Otros aspectos de la vida a bordo}

La extraordinaria preservación de los restos orgánicos que posee el sitio Swift permitió estudiar algunos temas relacionados con este tipo de evidencia arqueológica. Uno de ellos es el de los alimentos y bebidas almacenados y eventualmente consumidos a bordo de la nave.

Las provisiones habituales para este tipo de viajes incluían carne de res y de cerdo, pan, queso, arvejas, avena, mantequilla y cerveza, pero en ocasiones también podía haber bacalao seco, pasas de uva u otro fruto, brandy, harina, vinagre, grasa bovina (denominada suet) y aceite en reemplazo de mantequilla para las naves comisionadas a zonas tropicales (RODGER, 1986: 83). Algunos de estos productos incluso están consignados en distintos contextos relacionados con la Swift previamente a su viaje a Puerto Egmont (ADM 111/65, 1769; ADM 1/1789, 1770).

Aún no se excavaron las bodegas y ciertos sectores de la cocina que podrían almacenar alimentos; sin embargo, en el sector ocupado por los oficiales se hallaron restos de pasas de uva, semillas de zapallo, y condimentos como nuez moscada, dos especies de pimienta y mostaza (imagen 7). Todos estos elementos fueron encontrados en distintos tipos de recipientes, en su mayoría de vidrio (RODRÍGUEZ, 2011; PICCA, 2011).

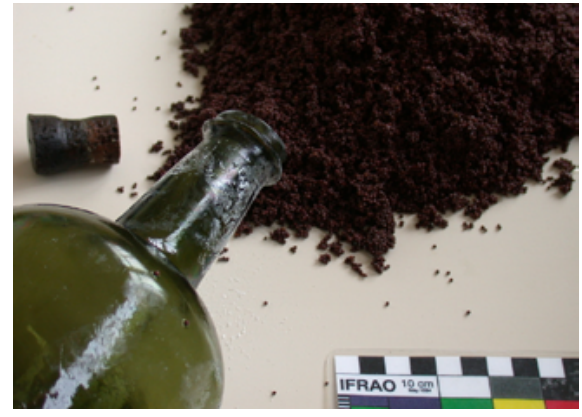

7. Granos de mostaza hallados en el interior de una botella de vidrio cilíndrica
Los restos faunísticos son muy escasos, pero ello es de esperarse a bordo de cualquier nave en la cual los desperdicios se arrojan al mar y la carne seca o salada se transportaba ya sin hueso. Probablemente este era el caso en la corbeta Swift, aunque debería ampliarse la excavación en el sector de la cocina para llegar a conclusiones adecuadamente sustentadas.

Un hallazgo particularmente interesante consiste en restos de huevos de pingüino Rey (FRERE, comunicación personal, 2000). Es lógico pensar que la recolección de este recurso permitía complementar la limitada dieta basada en las provisiones del Almirantazgo con una importante fuente de proteínas. 
Con respecto a las bebidas, la aparición de varias botellas cerradas con su correspondiente tapón de corcho permitió realizar análisis químico del líquido contenido en su interior. Una de ellas, del tipo de las clásicas botellas cilíndricas conocidas como "botellas de vino" y realizadas en vidrio de color verde o marrón, contenía un vino blanco tipo jerez (INFORME, 1982). Es posible que esto refleje la provisión de vino de Madeira mencionada para la Swift en alguna de las fuentes (ADM 3/77).

Por su parte, las llamadas habitualmente "botellas de ginebra", también de vidrio oscuro pero de sección cuadrada y en ocasiones de considerable mayor tamaño (imagen 8), habían sufrido filtraciones de agua de mar en su interior. Consiguientemente solo pudo establecerse que contenían algún tipo de bebida alcohólica, sin poder especificar el tipo (REPORT, 2001).

Otros recipientes que se hallaron cerrados corresponden a lo que probablemente era el arcón medicinal perteneciente al cirujano de a bordo. En este caso se trata de frascos pequeños, casi todos ellos de vidrio (imagen 9). Los análisis químicos efectuados sobre el contenido revelaron la presencia de sustancias que se habrían utilizado para el tratamiento de distintas enfermedades y dolencias. Uno de los recipientes encontrados dentro de este arcón contenía mercurio, habitualmente empleado en esa época para combatir la sífilis; otro contenía un extracto de antraquinona, el cual se consideraba apropiado para el tratamiento del escorbuto. También se halló copal, sustancia recomendada para el tratamiento de afecciones de la garganta y las encías, así como para restablecer la actividad del sistema nervioso central tras haber sufrido traumas severos, como quemaduras (ELKIN; EDWARDS; MAIER, 2012).

\section{Procesos de formación del sitio}

En toda investigación arqueológica resulta de gran utilidad comprender los distintos procesos - de índole natural y cultural- que han hecho que los restos se presenten tal como lo están al momento del hallazgo. En un ambiente como el que rodea al sitio Swift, altamente dinámico, uno de los aspectos tratados es el modo en que factores tales como la acción de las corrientes pudo haber modificado la disposición de los materiales o incluso haber causado la total desaparición de algunos de ellos. Las grandes algas del género Macrocystis también juegan un rol importante: al quedar atrapadas entre cuadernas u otras partes del barco que sobresalen del fondo, ejercen considerable tracción en los momentos de mayor corriente.

Parte de la investigación del sitio Swift fue entonces consagrada al monitoreo de todo este tipo de agentes. Entre otras cosas pudo observarse que las partes del sitio que más sobresalen del lecho marino se encuentran en una situación bastante vulnerable y requerirían la toma de medidas orientadas a su mejor preservación.

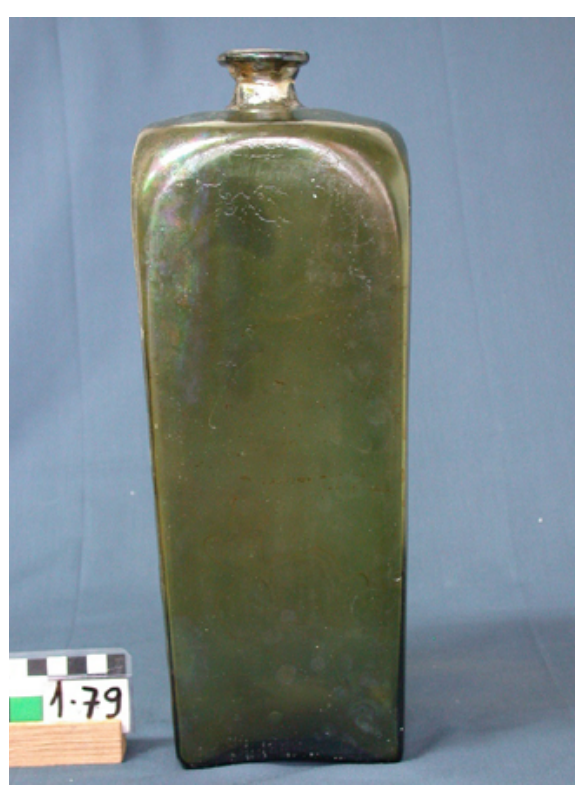

8. Una de las botellas de vidrio conocidas habitualmente como "de ginebra". En el sitio Swift algunas botellas de este tipo se hallaron cerradas con tapón de corcho

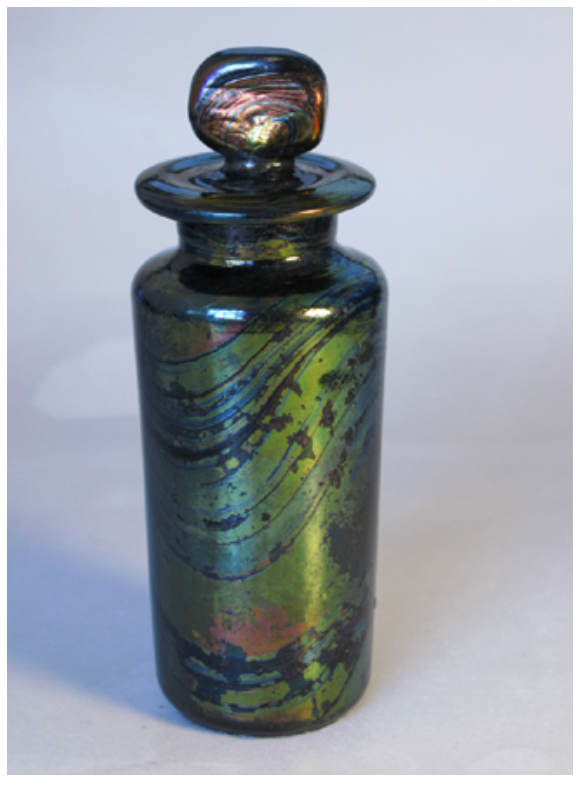

9. Uno de los frascos medicinales hallados dentro de un arcón de madera que habría pertenecido al cirujano de a bordo, con un tamaño aproximado de $11 \mathrm{~cm}$ de altura y $4 \mathrm{~cm}$ de diámetro 
También hay organismos, como los pequeños moluscos conocidos como teredos, que pueden perforar la madera causando un importante daño estructural. En general se ha considerado que los teredos no se desarrollan en aguas frías, pero se ha podido constatar que ciertos componentes de madera del pecio Swift (en particular aquellos que no están protegidos por una capa de sedimento) han sufrido un daño considerable como resultado de la acción de este tipo de organismos (BASTIDA; ELKIN; GROSSO et ál., 2002; BASTIDA; GROSSO; ELKIN, 2008; GROSSO, 2008).

Más allá del caso específico del pecio Swift, estos estudios permiten predecir procesos similares en otros pecios ubicados en ambientes marinos comparables y orientar políticas de investigación y gestión.

\section{Los restos humanos}

Durante la temporada de excavación del verano 2005-2006, centrada en la cabina del capitán Farmer, se produjo un hallazgo inesperado: un zapato de cuero con huesos humanos en su interior. Se encontraba adyacente a una tibia y peroné que comenzaban a asomar del sedimiento, sugiriendo que el resto del esqueleto se encontraba enterrado.

La razón de la sorpresa radicó en que, como se indicó, los documentos históricos señalaban que solo tres personas habían fallecido durante el naufragio: dos soldados pertenecientes a la dotación de marines a bordo (Robert Rusker, de 21 años, y John Ballard, de 23), y el cocinero, de nombre Richard Griffiths y 30 años de edad (ADM 33-688, 1770; ADM 5304, 1770; GOWER, 1803). Las fuentes también consignaban que uno de estos individuos -sin especificar cuál de ellos- fue hallado y enterrado en la costa. Podía deducirse que los cuerpos de los otros dos nunca aparecieron, y era razonable pensar que habían sido llevados por el agua.

En virtud de la naturaleza de este descubrimiento, la primera decisión fue suspender la excavación en el sector correspondiente cubriéndolo con bolsas de arena para su mejor protección. El paso siguiente fue reportar el hallazgo tanto a las autoridades argentinas correspondientes como a la Embajada Británica en Buenos Aires, a fin de consensuar el procedimiento a seguir. También se mantuvo la mayor discreción posible con los medios de prensa, intentando que prevaleciera una actitud de respeto por el cuerpo humano hallado, más allá de los siglos transcurridos desde su muerte.

Tras una serie de conversaciones e intercambio de ideas entre las distintas partes interesadas en el caso se acordó continuar con la recuperación del esqueleto para su estudio, tras lo cual se le daría sepultura en el sector británico del cementerio de la Chacarita en la ciudad de Buenos Aires (ELKIN, 2009; UNDERWOOD, 2007). 
El esqueleto humano hallado en el sitio Swift fue estudiado desde el punto de vista bioantropológico (BARRIENTOS; BÉGUELIN; BERNAL et ál., 2011) y también en función de los materiales adheridos y/o asociados al cuerpo, consistentes en restos de la indumentaria que vestía en el momento de morir (MAIER; PARERA; GÓMEZ, 2011). También se tomaron muestras dentales y óseas para eventuales estudios futuros.

Los análisis bioantropológicos revelaron que el cuerpo corresponde a un hombre joven, de alrededor de 25 años de edad, probablemente diestro y que gozaba de buena salud general, con la sola excepción de cálculo dental y unas pocas caries. También pudo comprobarse que su muerte no fue causada por un trauma, lo que hace pensar que debió haberse ahogado por haber quedado atrapado dentro del barco.

El estado de preservación de los huesos permitió la extracción exitosa de ADN. Sin embargo, por tratarse del tipo mitocondrial, ello dificulta enormemente el rastreo genealógico ya que éste se transmite por línea femenina y han transcurrido numerosas generaciones desde 1770 hasta la actualidad. La estimación de edad en base a los huesos, por su parte, tampoco permite asociarlo con alguna de las personas fallecidas en el naufragio debido a una cuestión estadística.

El análisis de restos de tela asociados a los huesos sí permitió establecer algo muy significativo: que la persona vestía una chaqueta de lana teñida de rojo con una sustancia obtenida de la planta conocida como Rubia roja o madder (Rubia tinctorum). Ello permitió inferir que esta prenda formaba parte del uniforme asignado a los soldados del grupo de marines, inferencia reforzada por la presencia de algunos botones, hebillas y fragmentos de polainas, que también habrían sido de un uniforme y no de la indumentaria utilizada por los marineros comunes (DE ROSA; CIARLO; SVOBODA, 2009).

A partir de todas las líneas de evidencia expuestas se concluye que el cuerpo hallado en el sitio Swift corresponde o bien a Robert Rusker o bien a John Ballard, es decir a uno de los dos infantes de marina reportados como desaparecidos en el naufragio.

\section{CONSIDERACIONES FINALES}

Quisiéramos concluir este trabajo haciendo referencia a otros aspectos del proyecto Swift, no menos importantes que la investigación arqueológica. De hecho uno de los grandes pilares del proyecto está representado por el Museo Mario Brozoski, el cual, como ya se indicó, ha tenido a su cargo desde las primeras intervenciones en el sitio la conservación y guarda de la colección completa de artefactos, además de destinar una de sus salas a una exhibición permanente. 


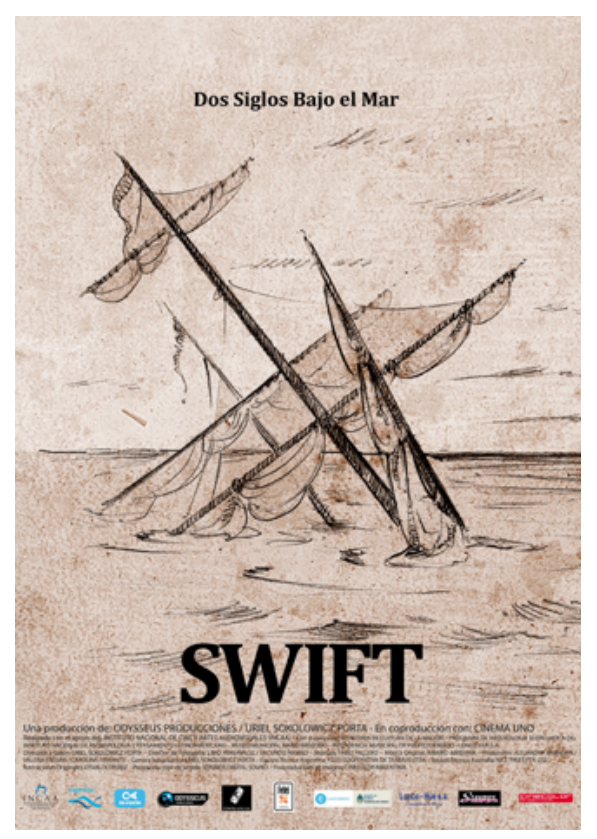

10. Afiche promocional del film Swift: Dos siglos bajo el mar (Director: Uriel Sokolowicz Porta)
Este pequeño museo, con la extraordinaria colección arqueológica que alberga, podría constituir un importante atractivo turístico en la región patagónica. Sin embargo, distintos factores reducen las posibilidades de que ello se concrete. Uno de ellos es la localización geográfica de Puerto Deseado, ya que se encuentra a $120 \mathrm{~km}$ de la principal ruta costera patagónica, carece de aeropuerto de uso civil, y los servicios de transporte terrestre son limitados (ELKIN; CAFFERATA, 2001). Por otra parte, al tratarse de un barco inglés, la conexión emocional o el sentido de identidad que posee la población local respecto a él son muy reducidos. Ello se acentúa por la controversia relativa a la soberanía de las islas Malvinas que ha enfrentado históricamente a Argentina y Gran Bretaña, alcanzando incluso dimensiones bélicas en un momento casualmente coincidente con el descubrimiento del pecio.

A pesar de lo expresado en el párrafo precedente, nuestro equipo de investigación y varios colaboradores -incluyendo individuos e instituciones de Puerto Deseado- han destinado muchos esfuerzos a actividades de difusión, transferencia y puesta en valor del Proyecto Swift. Ello ha incluido numerosas charlas públicas y entrevistas periodísticas, la organización de exhibiciones temporales sobre el sitio Swift fuera de Puerto Deseado, incluyendo una importante muestra en la ciudad de Buenos Aires, y hasta la elaboración de un largometraje documental que narra la historia del descubrimiento del pecio y el proyecto arqueológico generado a partir del mismo (imagen 10). Este fue realizado en el año 2010 con el apoyo del Instituto Nacional de Cine y Artes Audiovisuales (INCAA) de Argentina. Ejemplos como dicho largometraje, la exitosa muestra temporal efectuada en Buenos Aires, y la periódica cobertura del proyecto Swift en medios de alcance internacional -más aún hoy en día gracias a los múltiples recursos de internet-, evidencian que el interés en este proyecto fuera del ámbito académico bien puede trascender la comunidad local deseadense, la región patagónica y la misma República Argentina.

Quizás la Convención de la UNESCO para la Protección del Patrimonio Cultural Subacuático (CONVENCIÓN, 2001), de la cual la Argentina es Estado Parte, también genere un efecto positivo sobre el sitio Swift, directa o indirectamente. Esta convención, que a la fecha ha sido ratificada por más de 50 naciones, ya está contribuyendo a la mayor toma de conciencia acerca del valor de los naufragios históricos y otros vestigios culturales sumergidos. Este valor debe ser considerado tanto desde el punto de vista patrimonial como también en función de los beneficios económicos asociados que pueden generarse precisamente si se mantiene la integridad de dicho patrimonio, como puede ser el turismo. Asimismo, pone el acento en la cooperación internacional. Cuando se trata de un pecio, los principales estados relacionados con él suelen ser el estado de pabellón y el estado en cuyas aguas jurisdiccionales se encuentra actualmente el sitio. En el caso de la corbeta Swift, es de destacar la excelente relación que siempre tuvo lugar entre las auto- 
ridades argentinas y británicas en este sentido, dejando de lado eventuales disputas acerca de la titularidad del pecio y aunando esfuerzos para preservarlo lo mejor posible dentro de las capacidades de cada parte interesada.

Retornando por último a la investigación arqueológica, tras la publicación del libro al que ya hemos hecho referencia y que compila el trabajo efectuado a lo largo de más de 15 años (ELKIN; MURRAY; BASTIDA et ál., 2011) la excavación del sitio Swift ha entrado en un impasse. La razón principal es la falta de condiciones apropiadas en Puerto Deseado como para garantizar la conservación de nuevos materiales procedentes del pecio.

Sin embargo, las autoridades locales ya han expresado la intención de implementar las medidas necesarias para poder dar inicio a una nueva etapa de excavación e investigación arqueológica en el sitio, trabajando de manera mancomunada con el equipo del PROAS. De ser así, se continuará generando un valioso conocimiento acerca de esta pequeña nave de guerra inglesa del siglo XVIII que constituye un auténtico tesoro arqueológico. 


\section{BIBLIOGRAFÍA}

- BARRIENTOS, G.; BÉGUELIN, M.; BERNAL, V. et ál. (2011) Estudio bioarqueológico del esqueleto recuperado en la corbeta británica del siglo XVIII HMS Swift (Puerto Deseado, Santa Cruz, República Argentina). En ELKIN, D.; MURRAY, C.; BASTIDA, R. et ál. El naufragio de la HMS Swift (1770). Arqueología marítima en la Patagonia. CD Rom: Estudios especializados. Buenos Aires: Vázquez Mazzini Editores, 2011, pp. 1-26

- BASTIDA, R; GROSSO, M.; ELKIN, D. (2008) The role of benthic communities and environmental agents in the formation of underwater archaeological sites. En LESHIKAR-DENTON, M. E.; LUNA ERREGUERENA, P. (ed.) Underwater and Maritime Archaeology in Latin America and the Caribbean. Walnut Creek, California: Left Coast Press, 2008, pp. 173-185

- BASTIDA, R; ELKIN, D.; GROSSO, M. (2010) Enfoques interdisciplinarios para el estudio de procesos naturales de formación de sitios arqueológicos subacuáticos: investigaciones en el marco del proyecto Swift (provincia de Santa Cruz, Argentina). En OLIVA, F.; GRANDIS; N. (DE); RODRÍGUEZ, J. (comp.) Arqueología argentina en los inicios de un nuevo siglo, tomo III. Rosario, Santa Fe: Laborde Editor, 2010, pp. 269-283

- BASTIDA, R.; ELKIN, D.; GROSSO, M. et ál. (2002) La corbeta de guerra inglesa HMS Swift (1770): un caso de estudio sobre los efectos del biodeterioro en el patrimonio cultural subacuático de la Patagonia. En Actas de las Jornadas Científico Tecnológicas sobre Prevención y Protección del Patrimonio Cultural Iberoamericano del Biodeterioro Ambiental. La Plata, Buenos Aires: CYTED, LEMIT, CIDEPINT, Universidad Tecnológica Nacional, 2002, pp. 119-143

- CIARLO, N. C. (2011) Arqueometalurgia de un sitio de naufragio del siglo XVIII: la corbeta de guerra HMS Swift (1770), Puerto Deseado (provincia de Santa Cruz). Tesis de Licenciatura en Ciencias Antropológicas, Facultad de Filosofía y Letras, Universidad de Buenos Aires. MS en archivo: Programa de Arqueología Subacuática, Instituto Nacional de Antropología y Pensamiento Latinoamericano, Buenos Aires (documento inédito)

- CONVENCIÓN para la Protección del Patrimonio Cultural Subacuático (2001). París: UNESCO, 2001

- DELLINO-MUSGRAVE, V. (2005) British Identities Through Pottery in Praxis. The Case Study of a Royal Navy Ship in the South Atlantic. Journal of Material Culture, 10 (3), 2005, pp. 219-243

- DELLINO-MUSGRAVE, V. (2006) Maritime Archaeology and Social Relations. British Action in the Southern Hemisphere. New York, NY: Springer, 2006 (The Springer series in Underwater Archaeology)

- DE ROSA, H.; CIARLO, N. C.; SVOBODA, H. (2009)
Estudio sobre botones de peltre hallados en la corbeta HMS Swift (1770). En PALACIOS, M. O.; VÁZQUEZ, C.; PALACIOS, T. et ál. (ed.) Arqueometría Latinoamericana: Il Congreso Argentino y I Latinoamericano, vol. 1. Buenos Aires: Comisión Nacional de Energía Atómica, 2009, pp. 227-232

- ELKIN, D. (1997) Proyecto Arqueológico Swift. MS en archivo, Instituto Nacional de Antropología y Pensamiento Latinoamericano (documento inédito)

- ELKIN, D. (2003) Arqueología marítima y patrimonio cultural subacuático en Argentina. El trabajo actual desarrollado por el Instituto Nacional de Antropología y Pensamiento Latinoamericano. En Protección del Patrimonio Cultural Subacuático en América Latina y el Caribe. La Habana: UNESCO-Oficina regional de Cultura para América Latina y el Caribe, 2003, pp. 26-33

- ELKIN, D. (2009) Case Study: HMS Swift / Argentina. En Shared Heritage: Joint Responsibilities in the Management of British Warship Wrecks overseas [en línea] Reino Unido: English Heritage, University of Wolverhampton, 2009, pp. 8-19<https://content.historicengland.org.uk/imagesbooks/publications/management-of-british-warship-wrecksoverseas/shared-heritage-management-of-british-warshipwrecks-overseas.pdf/> [Consulta: 17/02/2016]

- ELKIN, D.; CAFFERATA, H. (2001) Underwater archaeology and cultural tourism-a mutual benefit proposal for Patagonia. The Bulletin of the Australasian Institute for Maritime Archaeology (AIMA Bulletin), vol. 25, 2001, pp. 8388

- ELKIN, D.; ARGÜESO, A.; GROSSO, M. et ál. (2007) Archaeological research on HMS Swift: a British Sloopof-War lost off Patagonia, Southern Argentina, in 1770. International Journal of Nautical Archaeology, 36 (1), 2007. pp. 32-58

- ELKIN, D.; ARGÜESO, A. (2010) Aportes TeóricoMetodológicos para arqueología náutica de momentos históricos. El caso de la corbeta de guerra HMS Swift (Puerto Deseado, Santa Cruz). En OLIVA, F.; GRANDIS, N. DE; RODRÍGUEZ, J. (comp.) Arqueología argentina en los inicios de un nuevo siglo, tomo III. Rosario, Santa Fe: Laborde Editor, 2010, pp. 337- 345

- ELKIN, D.; MURRAY, C.; BASTIDA, R. et ál. (2011) El naufragio de la HMS Swift (1770). Arqueología marítima en la Patagonia. Buenos Aires: Vázquez Mazzini Editores, 2011

- ELKIN, D.; EDWARDS, H. G. M.; MAIER, M. S. (2012)

The Application of Analytical Archaeometry in Underwater Cultural Heritage-A Case Study from Patagonia, Argentina. En EDWARDS, H.; VANDENABEELE, P. (ed.) Analytical Archaeometry. Cambridge: Royal Society of Chemistry, 2012, pp. 546-562 
- GOWER, E. (1803) An Account of the Loss of His Majesty's Sloop'Swift', in Port Desire, on the Coast of Patagonia, on the $13^{\text {th }}$ of March, 1770; and of the other Events Which Succeeded, in a Letter to a Friend. Londres: Winchester and Son, 1803

- GROSSO, M. (2008) Arqueología de naufragios: estudio de procesos de formación naturales en el sitio HMS Swift (Puerto Deseado, Santa Cruz) En Tesis de Licenciatura, vol. 2. Departamento de Ciencias Antropológicas. CD Rom. Buenos Aires: Facultad de Filosofía y Letras de la Universidad de Buenos Aires, 2008

- GRosso, M. (2011) Estudios de cultura material en sitios históricos de naufragio en el litoral patagónico. El uso de la madera en artefactos del barco británico HMS Swift (siglo XVIII). Tesis doctoral inédita, Facultad de Filosofía y Letras, Universidad de Buenos Aires. MS en archivo: Programa de Arqueología Subacuática, Instituto Nacional de Antropología y Pensamiento Latinoamericano, Buenos Aires

- HAWKINS, J. (1999) Staffordshire Engine Turned Pottery, 1760 - 1780. Bulletin of the Society of Ornamental Turners, vol. 20,1999, n. ${ }^{\circ} 100$ [en línea] <http://www.jbhawkinsantiques. com/uploads/articles/StaffordshireEngineTurnedPottery1. pdf> [Consulta: 17/02/2019]

- INFORME sobre muestras de materiales hallados en la ría Deseado (1982) Dirección Nacional de Química MS en archivo: Programa de Arqueología Subacuática, Instituto Nacional de Antropología y Pensamiento Latinoamericano, Buenos Aires

- MAIER, M. S.; PARERA S. D.; GÓMEZ, B. A. (2011) Identificación de fibras textiles adheridas a un esqueleto humano proveniente de un sitio arqueológico subacuático de Patagonia. En ELKIN, D.; MURRAY, C.; BASTIDA, R. et ál. El naufragio de la HMS Swift (1770). Arqueología marítima en la Patagonia. Buenos Aires: Vázquez Mazzini Editores, 2011, pp. 125-129 (CD Rom: Estudios especializados)

- MURRAY, C.; ELKIN, D.; VAINSTUB, D. (2002-2003) The Sloop-of-War HMS Swift: An Archaeological Approach. En TRACY, N. (ed.) The Age of Sail. Londres: Conway Maritime Press, 2002-2003, pp. 101-115

- PICCA, P. (2011) Identificación taxonómica de algunos restos botánicos de especies de interés alimenticio en el sitio de naufragio de la HMS Swift (1770). En ELKIN, D.; MURRAY, C.; BASTIDA, R. et ál. El naufragio de la HMS Swift (1770). Arqueología marítima en la Patagonia. Buenos Aires: Vázquez Mazzini Editores, 2011, pp. 139145 (CD Rom: Estudios especializado)

- REPORT on the content of bottles from the HMS Swift archaeological site (2001) UDV Laboratory Harlow MS en archivo: Programa de Arqueología Subacuática, Inapl, Buenos Aires (documento inédito)
- RODGER, N. A. M. (1986) The Wooden World. An anatomy of the Georgian Navy. New York-London: W. Norton \& Co, 1986

- RODRÍGUEZ, M. F. (2011) Macrovestigios vegetales recuperados en el sitio arqueológico HMS Swift (1770). En ELKIN, D.; MURRAY, C.; BASTIDA, R. et ál. El naufragio de la HMS Swift (1770). Arqueología marítima en la Patagonia. Buenos Aires: Vázquez Mazzini Editores, 2011 (CD Rom: Estudios especializados)

- UNDERWOOD, C. (2007) The interment of an 18th Century British Private Marine in Argentina. Nautical Archaeology, (3):10, 2007, p. 10

- VÁZQUEZ, C.; BOEYKENS, S.; ELKIN, D. (2010) The Use of Total Reflection X-Ray Fluorescence in an Underwater Archaeology Case Study. Technical Briefs in Historical Archaeology, 5, 2010, pp. 10-15

\section{DOCUMENTOS HISTÓRICOS}

The National Archives, Inglaterra:

- ADM 111/65 Victualling board and committees, 1769

- ADM 1/1789 Captain's Letters: George Farmer, 17/11/1769, 19/02/1770 y 22/09/1770

- ADM 1//5304 Courts Martial: Loss of HMS Swift, 29/09/1770

- ADM 3/77 Board's minutes, 16/08/1769 y 19/10/1769

- ADM 33/688 Ships' muster books: Swift,1769-1770

National Maritime Museum, Inglaterra:

- NMM ADM 180/3 Progress and Dimension Books: Swift 1762-1769

- NMM ZAZ 4672 Lines \& profile, Swift and Vulture, 1763

- NMM ZAZ 4724 Decks, Vulture Class, 1762 